\title{
Additional lymphadenectomy might not improve survival of patients with resectable metastatic colorectal adenocarcinoma of T4 stage, proximal location, poor/undifferentiation, or N3/N4 stages: a large population-based study.
}

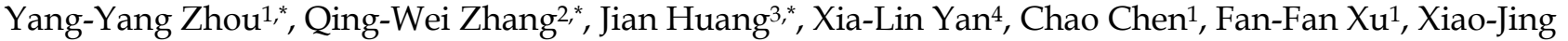 \\ $\mathrm{Du}^{1}$, Rong Jin ${ }^{1,5}{ }^{\circledR}$ \\ 1. Department of Gastroenterology, The First Affiliated Hospital of Wenzhou Medical University, Wenzhou325000, Zhejiang, China. \\ 2. Division of Gastroenterology and Hepatology, Key Laboratory of Gastroenterology and Hepatology, Ministry of Health, Renji Hospital, School of Medicine, \\ Shanghai Jiao Tong University; Shanghai Institute of Digestive Disease;145 Middle Shandong Road, Shanghai 200001, China. \\ 3. Department of Gastroenterology, Yuyao People's Hospital of Zhejiang Province, The Affiliated Yangming Hospital of Ningbo University, Ningbo 315400, \\ Zhejiang, China. \\ 4. Department of Surgery, Ruijin Hospital, Shanghai Jiao Tong University School of Medicine, No. 197, Ruijin 2nd Rd, Shanghai 200025, China. \\ 5. Department of Epidemiology, The First Affiliated Hospital of Wenzhou Medical University, Wenzhou325000, Zhejiang, China.
}

*Equal contributions

$\triangle$ Corresponding author: Rong Jin, Department of Gastroenterology, Department of Epidemiology. The First Affiliated Hospital of Wenzhou Medical University, Wenzhou325000, Zhejiang, China. Email: jinrongjiri@163.com; Tel:+86 57786689984

(c) Ivyspring International Publisher. This is an open access article distributed under the terms of the Creative Commons Attribution (CC BY-NC) license (https://creativecommons.org/licenses/by-nc/4.0/). See http://ivyspring.com/terms for full terms and conditions.

Received: 2018.01.01; Accepted: 2018.05.15; Published: 2018.06.14

\begin{abstract}
This study was performed to evaluate the prognostic effect of lymphadenectomy on outcomes in patients with resectable metastatic colorectal adenocarcinoma (mCRC). We selected patients with mCRC from 2004 to 2013 from Surveillance, Epidemiology, and End Results Program (SEER) database. Kaplan-Meier analysis, univariate Cox regression and multivariate Cox regression analysis were performed to assess the clinical value of lymphadenectomy on overall survival (OS) and cause-specific survival (CSS) of patients with resectable mCRC. A total 24178 eligible patients were included, 23056 (95.36\%) of which received lymphadenectomy. Results showed that lymphadenectomy was an independent protective factor for survival of patients with $\mathrm{mCRC}$ overall [OS (HR: 0.86, 95\%Cl: 0.79-0.93, $\mathrm{P}=0.002$ ) and CSS (HR: 0.85, 95\%Cl: 0.78-0.93, $\mathrm{P}<0.001)$ ]. Further analysis showed that lymphadenectomy improved survival of patients with Tl stage [OS (HR: 0.51, 95\%Cl: 0.39-0.66, P<0.001); CSS (HR: 0.48, 95\%Cl: 0.36-0.65, P<0.001)], distal [OS (HR: 0.65, 95\%Cl: 0.56-0.75, P<0.001); CSS (HR: 0.65, 95\%Cl: 0.65-0.75, P<0.001)], rectal [OS (HR: 0.60, 95\%Cl: $0.52-0.70, \mathrm{P}<0.001)$; CSS (HR: $0.59,95 \% \mathrm{Cl}: 0.51-0.69, \mathrm{P}<0.001)]$, well/moderately differentiated [OS (HR: 0.62, 95\%Cl: 0.56-0.70, P<0.001); CSS (HR: 0.62, 95\%Cl: 0.55-0.69, $\mathrm{P}<0.001$ )], Nl stage [OS (HR: 0.76, 95\% Cl: 0.67-0.85, P<0.001); CSS (HR: 0.74, 95\%Cl: 0.65-0.84, $\mathrm{P}<0.001$ )] and N2 stage [OS (HR: 0.63, 95\%Cl: 0.54-0.74, P<0.001; CSS (HR: 0.65, 95\%Cl: 0.55-0.77, $\mathrm{P}<0.001)) \mathrm{mCRC}$. While lymphadenectomy might not improve survival of patients with T4 stage, proximal, poor or undifferentiated, N3 and N4 stage mCRC. In general, Additional lymphadenectomy was suggested for patients with mCRC overall. However, lymphadenectomy might not improve survival of patients with $\mathrm{mCRC}$ of higher malignancy tendency, such as T4 stage, proximal location, poor or undifferentiation, N3 and N4 stages.
\end{abstract}

Key words: Metastatic colorectal adenocarcinoma, SEER, Lymphadenectomy, Overall survival, Cause-specific survival 


\section{Introduction}

Colorectal adenocarcinoma (CRC) is the second leading cause of death due to cancer in the United States. According to expected number of new cancer cases and deaths in the United States, around 135,000 new cases of colorectal adenocarcinoma and 50,200 estimated deaths occur in 2017 [1]. Surgery is the foundation stone of therapy for CRC, and around 80 percent of patients have tumors that, at diagnosis, can be resected with curative intention, and chemotherapy is used most commonly in the adjuvant setting [2, 3]. The five-year overall survival for patients with localized, regional, and metastatic colon or rectal cancer is $91 \%, 72 \%$, and $13 \%$ [1]. Thus, it is of great significance to improve the survival rate of patients with metastatic colorectal cancer.

As for CRC patients of any $\mathrm{T}$ stage, any $\mathrm{N}$ stage, and resectable synchronous metastases, synchronous resection or local therapy for metastases and resection of primary lesion are preferred according to National Comprehensive Cancer Network (NCCN) Guidelines Version1.2017 [4, 5]. When it comes to excision of lymph nodes, the International Union Against Cancer and the American Joint Committee on Cancer (AJCC) have recommended evaluation of at least 12 lymph nodes to confirm node-negative colon or rectal cancer [6]. Le Voyer et al. illustrated that an increase in the number of lymph nodes examined was significantly associated with improved survival for patients of colon cancer [7]. In addition, a more recent analysis of patients with stage I or stage II rectal cancer from SEER database revealed that survival improved with greater numbers of lymph nodes dissection [6]. However, two studies above were restricted to TNM from stage I to stage III. As a matter of fact, approximately 60 percentage of colorectal cancer patients would finally develop metastases [8-10], and the overall survival of mCRC was poor. The poor outcomes may be induced to some extent by the wrong choice of treatment including operation methods and the optimal sequencing of adjuvant chemotherapy, radiation therapy, which was on the debate and contradictory [3, 11]. Whether surgery should be performed to the primary CRC and lymph nodes is one of questions which should be answered. In previous study, we conducted large-population based retrospective study to analyze whether primary rectal cancer dissection improved survival of patients with metastatic rectal cancer and found primary rectal cancer dissection did contribute to better survival [12]. With respect to lymph node dissection, the present studies even lacked consensus on whether lymphadenectomy improve survival of resectable mCRC. A retrospective cohort study just showed that there was no survival benefit from the use of routine lymph node dissection of liver metastasis for mCRC [13]. While Bradatsch et al. revealed that lymph node dissection of metastases could provide a prognostic tool to better further treatment [14]. All of them paid attention to lymph node dissection of distal metastases. However, whether lymphadenectomy is needed for primary mCRC has not yet been clearly illuminated. In this study, we conducted a study using Surveillance, Epidemiology and End Results Program (SEER) database involved a large population with resectable mCRC from 2004 to 2013 to assess the prognostic value of lymph node dissection.

\section{Materials and methods}

\section{Data source}

Data was acquired from SEER datasets to identify adult patients with mCRC in 2004-2013. We gathered information on lymph node dissection, race, sex, age, grade, tumor (T) stage, lymph node metastasis $(\mathrm{N})$, chemotherapy, radiation, location of carcinoma.

\section{Patients}

Eligibility for enrollment into the study was established using the following inclusion criteria: (1) the primary cancer site located in the colorectum using the code C18.0-18.9 and C20.9; (2) the histological subtypes of CRC confirmed to be adenocarcinoma, mucinous adenocarcinoma, signet-ring cell carcinoma using the variable "Histologic Type ICD-0-3" code 8140, 8144-45, $8210-11,8213,8220-21,8255,8260-63$ and 8323 for colorectal adenocarcinoma, $8480-81$ for mucinous adenocarcinoma and 8490 for signet-ring cell carcinoma; (3) age $\geq 18$ years; (4) histologically-confirmed colorectal adenocarcinoma; (5) clinically-documented metastasis; (6) only one tumor or the first tumor of primary tumors; (7) treatment with primary tumor surgery; (8) survival time $\geq 3$ month; (9) complete information on grade, $\mathrm{T}$ stage, tumor location, race, radiation and lymph node resection. We have gotten access to SEER database for only research purpose using the private SEER ID (zhangqw).

\section{Variables}

The main variable interested us was lymph node dissection. According to the status of whether lymphadenectomy was performed among patients with primary $\mathrm{mCRC}$ dissection, patients were divided into two groups: lymph node dissection group and non-lymph node dissection group. There were mainly three kinds of race classified in the study, namely the white race, the black and other race (containing American Indian, Asian and Pacific Islander). Age 
was divided into two parts: below 65 years old and at least 65 years old. Histology was classified as adenocarcinoma, mucinous adenocarcinoma and signet ring cell carcinoma. As for differentiation grades, well or moderately differentiated cancers were separated from poorly differentiated or undifferentiated carcinomas. $\mathrm{T}$ stage was categorized as T1, T2, T3 and T4 according to the AJCC Cancer Staging Manual (the 6th edition), and lymph node (N) stage was divided as N1, N2, N3 and N4 as well. Whether to receive adjuvant therapy such as chemotherapy or radiation was also considered in the study. Chemotherapy information for mCRC was classified as yes or no/unknown. Also consistent with chemotherapy information, radiation was reclassified as yes or no. Due to the difference of the anatomic characteristic, we classified tumor location as proximal colon, distal colon and rectal cancer.

\section{Statistical analysis}

In our study, OS and CSS were mainly considered for our primary outcomes. CSS was defined as death caused by mCRC. Visualization and calculation of survival rates were demonstrated using Kaplan-Meier analysis and log-rank test. Univariate and multivariate Cox regression analysis were

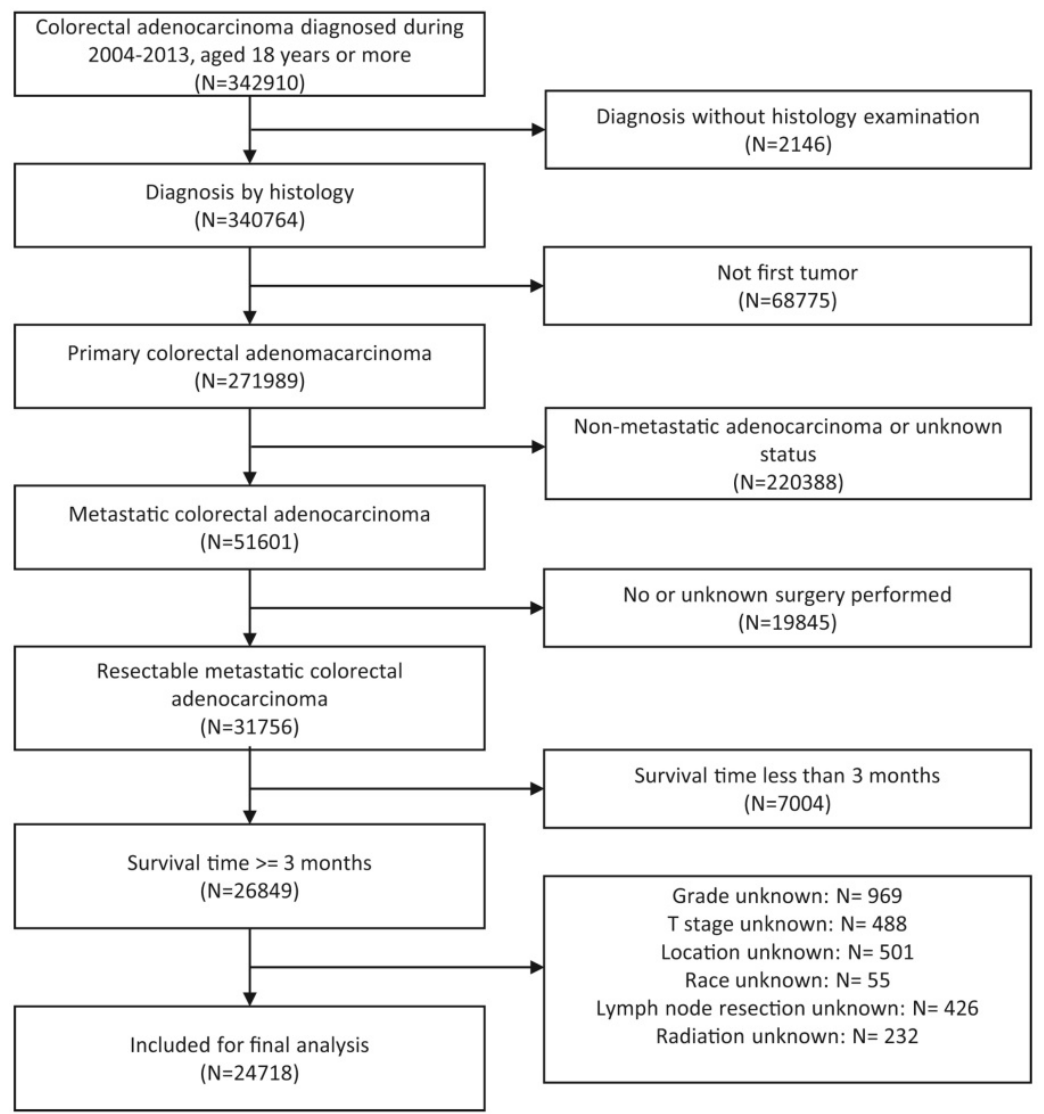

Figure 1. Flow chart of patients' cohort definition. With inclusion and exclusion criteria, final 24718 metastatic colorectal adenocarcinoma were identified from patients with initially identified 342910 colorectal adenocarcinoma diagnosed during 2004-2013. conducted to analyze affecting factors for OS and CSS of patients with primary mCRC resection. All data analyses were carried out with the Stata13.0 software and $\mathrm{R}$ software 3.3.3. P-value less than 0.05 was considered statistically significant. The results were shown with hazard ratios (HR) with 95\% confidence intervals (CIs)

\section{Results}

\section{Characteristics of patients with MCRC}

The details on participants' cohort definition were shown in flow chart (Figure 1). A total 24178 eligible patients with mCRC were identified, of which $23056(95.36 \%)$ received lymphadenectomy of primary tumor and $1122(4.64 \%)$ did not. The median follow-up time of the patients was 20 months (scope 3-119 months). The distribution of race, sex, age, chemotherapy, radiation and location had little difference between lymph node dissection group and non-lymph node dissection group. Of patients with lymph node dissection, $85.53 \%$ of them were adenocarcinoma, while just $66.04 \%$ of patients without lymph node dissection were adenocarcinoma. As for histological grade, patients in lymph node dissection group had a large proportion $(61.02 \%)$ of well or moderately differentiated carcinoma, while the proportion of poorly differentiated or undifferentiated disease were slightly higher in non-lymph node dissection group. With the respect of $\mathrm{T}$ stage, $\mathrm{T} 3$ and T4 accounted for most in both group. The distribution of $\mathrm{N}$ stage were slightly different in two group. Specifically, around $81.69 \%$ of patients with lymph node excision were in $\mathrm{N} 2$ and N3, whereas about $76 \%$ of patients without lymph node dissection were in N1 and N2. The detailed information for each clinical characteristic was described in the Table 1.

\section{Univariate and multivariate Cox regression analysis for evaluating the influence of lymph node dissection}

Kaplan-Meier curves for OS (Figure 2A) and CSS (Figure 2B) showed no significant difference (OS: $\mathrm{P}=0.26$, CSS: $\mathrm{P}=0.13$ ) between dissection of lymph nodes group and non-lymph nodes dissection group. Besides, univariate analysis of covariates for OS and CSS was carried out and details were shown in 
Table 2. Race, age, grade, $\mathrm{T}$ stage, $\mathrm{N}$ stage, histology, chemotherapy, radiation and location were significantly associated with OS and CSS. Yet, lymph node dissection [OS (HR: 1.04, 95\%CI: 0.97-1.12, $\mathrm{P}=0.268$ ) and CSS (HR: 1.06, 95\%CI: 0.98-1.15, $\mathrm{P}=0.135)]$ may not play an important role in improving survival outcomes using the univariate Cox regression analysis. Intriguingly, multivariate Cox regression analysis was inconsistent. In addition to race, age, grade, $\mathrm{T} 4$ stage, $\mathrm{N}$ stage, chemotherapy and location, lymph node excision was an independent prognostic risk factor of poor OS and CSS (Table 3). The results of multivariate Cox regression analysis showed that patients in lymph node dissection group had better OS (HR: 0.86, 95\% CI: $0.79-0.93, \mathrm{P}=0.002$ ) and CSS (HR: $0.85,95 \% \mathrm{CI}$ : $0.78-0.93, \mathrm{P}<0.001)$.

Table 1. Characteristics of patients with metastatic colorectal adenocarcinoma dissected of primary tumor included in this study.

\begin{tabular}{|c|c|c|c|}
\hline \multirow[t]{2}{*}{ Characteristics } & Total & $\begin{array}{l}\text { With lymph } \\
\text { node dissection }\end{array}$ & $\begin{array}{l}\text { Without lymph } \\
\text { node dissection }\end{array}$ \\
\hline & $24178(100 \%)$ & $23056(95.36 \%)$ & $1122(4.64 \%)$ \\
\hline \multicolumn{4}{|l|}{ Race } \\
\hline White & $\begin{array}{l}18629 \\
(77.05 \%)\end{array}$ & $17755(77.01 \%)$ & $874(77.90 \%)$ \\
\hline Black & $3352(13.86 \%)$ & $3201(13.88 \%)$ & $151(13.46 \%)$ \\
\hline Other race & 2197 (9.09\%) & $2100(9.11 \%)$ & $97(8.65 \%)$ \\
\hline \multicolumn{4}{|l|}{ Sex } \\
\hline Male & $\begin{array}{l}12549 \\
(51.90 \%)\end{array}$ & $12016(52.12 \%)$ & $533(47.50 \%)$ \\
\hline Female & $\begin{array}{l}11629 \\
(48.10 \%)\end{array}$ & $11040(47.88 \%)$ & $589(52.50 \%)$ \\
\hline \multicolumn{4}{|l|}{ Age } \\
\hline$<65$ & $\begin{array}{l}13650 \\
(56.46 \%)\end{array}$ & $12965(56.23 \%)$ & $685(61.05 \%)$ \\
\hline$>=65$ & $\begin{array}{l}10528 \\
(43.54 \%)\end{array}$ & $10091(43.77 \%)$ & $437(38.95 \%)$ \\
\hline \multicolumn{4}{|l|}{ Histology } \\
\hline Adenocarcinoma & $\begin{array}{l}20461 \\
(84.63 \%)\end{array}$ & $19720(85.53 \%)$ & $741(66.04 \%)$ \\
\hline $\begin{array}{l}\text { Mucinous } \\
\text { adenocarcinoma }\end{array}$ & $3134(12.96 \%)$ & $2812(12.20 \%)$ & $322(28.70 \%)$ \\
\hline Signet ring cell carcinoma & $583(2.41 \%)$ & $524(2.27 \%)$ & $59(5.26 \%)$ \\
\hline \multicolumn{4}{|l|}{ Grade } \\
\hline Well/moderate & $\begin{array}{l}14753 \\
(61.02 \%)\end{array}$ & $14215(61.65 \%)$ & $538(47.95 \%)$ \\
\hline Poorly/undifferentiated & $9425(38.98 \%)$ & $8841(38.35 \%)$ & $584(52.05 \%)$ \\
\hline \multicolumn{4}{|l|}{ T Stage } \\
\hline $\mathrm{T} 1$ & $451(1.87 \%)$ & $263(1.14 \%)$ & $188(16.76 \%)$ \\
\hline $\mathrm{T} 2$ & $698(2.89 \%)$ & $640(2.78 \%)$ & $58(5.17 \%)$ \\
\hline T3 & $\begin{array}{l}14353 \\
(59.36 \%)\end{array}$ & $13981(60.64 \%)$ & $372(33.16 \%)$ \\
\hline $\mathrm{T} 4$ & $8676(35.88 \%)$ & $8172(35.44 \%)$ & $504(44.92 \%)$ \\
\hline \multicolumn{4}{|l|}{ N Stage } \\
\hline N1 & $4808(19.89 \%)$ & $4175(18.11 \%)$ & $633(56.42 \%)$ \\
\hline N2 & $8067(33.37 \%)$ & $7851(34.05 \%)$ & $216(19.25 \%)$ \\
\hline N3 & $\begin{array}{l}11087 \\
(45.86 \%)\end{array}$ & $10983(47.64 \%)$ & $104(9.27 \%)$ \\
\hline N4 & $216(0.89 \%)$ & $47(0.20 \%)$ & $169(15.06 \%)$ \\
\hline \multicolumn{4}{|l|}{ Chemotherapy } \\
\hline Yes & $\begin{array}{l}17643 \\
(72.97 \%)\end{array}$ & $16884(73.23 \%)$ & $759(67.65 \%)$ \\
\hline No/unknown & $6535(27.03 \%)$ & $6172(26.77 \%)$ & $363(32.35 \%)$ \\
\hline \multicolumn{4}{|l|}{ Radiation } \\
\hline Yes & $2568(10.62 \%)$ & $2368(10.27 \%)$ & $200(17.83 \%)$ \\
\hline No & $\begin{array}{l}21610 \\
(89.38 \%)\end{array}$ & $20688(89.73 \%)$ & $922(82.17 \%)$ \\
\hline
\end{tabular}

\begin{tabular}{llll}
\hline Characteristics & Total & $\begin{array}{l}\text { With lymph } \\
\text { node dissection }\end{array}$ & $\begin{array}{l}\text { Without lymph } \\
\text { node dissection }\end{array}$ \\
\cline { 2 - 4 } & $24178(100 \%)$ & $23056(95.36 \%)$ & $1122(4.64 \%)$ \\
\hline Location & & & \\
Proximal colon & $9605(39.73 \%)$ & $9117(39.54 \%)$ & $488(43.49 \%)$ \\
Distal colon & $9501(39.30 \%)$ & $9215(39.97 \%)$ & $286(25.49 \%)$ \\
Rectum & $5072(20.98 \%)$ & $4724(20.49 \%)$ & $348(31.02 \%)$ \\
\hline
\end{tabular}

Table 2. Univariate Cox regression analysis for evaluating the influence of clinicalpathological characteristics on survival of patients with primary metastatic colorectal adenocarcinoma resection in SEER database.

\begin{tabular}{|c|c|c|c|c|}
\hline \multirow[t]{2}{*}{ Characteristics } & \multicolumn{2}{|l|}{ OS } & \multicolumn{2}{|l|}{ CSS } \\
\hline & HR $(95 \%$ CI) & $\mathrm{P}$ & HR $(95 \%$ CI) & $\mathrm{P}$ \\
\hline \multicolumn{5}{|l|}{ Lymph node dissection } \\
\hline No & 1 (Reference) * & & 1 (Reference) & \\
\hline Yes & $1.04(0.97-1.12)$ & 0.268 & $1.06(0.98-1.15)$ & 0.135 \\
\hline \multicolumn{5}{|l|}{ Race } \\
\hline White & 1 (Reference) & & 1 (Reference) & \\
\hline Black & $1.12(1.08-1.17)$ & $<0.001$ & $1.12(1.07-1.17)$ & $<0.001$ \\
\hline Other race & $0.92(0.87-0.97)$ & 0.002 & $0.90(0.85-0.95)$ & $<0.001$ \\
\hline \multicolumn{5}{|l|}{ Sex } \\
\hline Female & 1 (Reference) & & 1 (Reference) & \\
\hline Male & $0.99(0.96-1.02)$ & 0.700 & $0.99(0.96-1.02)$ & 0.468 \\
\hline \multicolumn{5}{|l|}{ Age } \\
\hline$<65$ & 1 (Reference) & & 1 (Reference) & \\
\hline$>=65$ & $1.49(1.44-1.53)$ & $<0.001$ & $1.41(1.37-1.46)$ & $<0.001$ \\
\hline \multicolumn{5}{|l|}{ Grade } \\
\hline Well/moderate & 1 (Reference) & & 1 (Reference) & \\
\hline Poorly/undifferentiated & $1.33(1.29-1.37)$ & $<0.001$ & $1.36(1.31-1.40)$ & $<0.001$ \\
\hline \multicolumn{5}{|l|}{ T stage } \\
\hline $\mathrm{T} 1$ & 1 (Reference) & & 1 (Reference) & \\
\hline $\mathrm{T} 2$ & $0.99(0.85-1.15)$ & 0.900 & $1.02(0.86-1.19)$ & 0.863 \\
\hline T3 & $1.32(1.17-1.49)$ & $<0.001$ & $1.44(1.26-1.64)$ & $<0.001$ \\
\hline $\mathrm{T} 4$ & $1.66(1.47-1.87)$ & $<0.001$ & $1.82(1.60-2.08)$ & $<0.001$ \\
\hline \multicolumn{5}{|l|}{ N stage } \\
\hline N1 & 1 (Reference) & & 1 (Reference) & \\
\hline N2 & $1.45(1.39-1.52)$ & $<0.001$ & $1.40(0.33-1.47)$ & $<0.001$ \\
\hline N3 & $1.90(1.81-1.98)$ & $<0.001$ & $1.89(1.80-1.97)$ & $<0.001$ \\
\hline N4 & $1.46(1.23-1.72)$ & $<0.001$ & $1.79(1.52-2.10)$ & $<0.001$ \\
\hline \multicolumn{5}{|l|}{ Histology } \\
\hline Adenocarcinoma & 1 (Reference) & & 1 (Reference) & \\
\hline Mucinous adenocarcinoma & $0.80(0.76-0.84)$ & $<0.001$ & $1.02(0.97-1.07)$ & 0.384 \\
\hline Signet ring cell carcinoma & $1.20(1.09-1.32)$ & $<0.001$ & $1.64(1.49-1.81)$ & $<0.001$ \\
\hline \multicolumn{5}{|l|}{ Chemotherapy } \\
\hline No/unknown & 1 (Reference) & & 1 (Reference) & \\
\hline Yes & $0.64(0.62-0.66)$ & $<0.001$ & $0.67(0.65-0.70)$ & $<0.001$ \\
\hline \multicolumn{5}{|l|}{ Radiation } \\
\hline No & 1 (Reference) & & 1 (Reference) & \\
\hline Yes & $0.76(0.72-0.80)$ & $<0.001$ & $0.76(0.73-0.81)$ & $<0.001$ \\
\hline \multicolumn{5}{|l|}{ Location } \\
\hline Proximal colon & 1 (Reference) & & 1 (Reference) & \\
\hline Distal colon & $0.80(0.77-0.82)$ & $<0.001$ & $0.80(0.77-0.82)$ & $<0.001$ \\
\hline Rectum & $0.72(0.69-0.75)$ & $<0.001$ & $0.72(0.69-0.75)$ & $<0.001$ \\
\hline
\end{tabular}

\section{Lymph node excision on survival of patients with $\mathrm{mCRC}$ in different $\mathrm{T}$ stages, tumor location, differentiation grades and $\mathbf{N}$ stages.}

To further demonstrate the prognostic role of lymph node excision in $\mathrm{mCRC}$, we performed Cox regression analysis of patients with primary mCRC resection according to different $\mathrm{T}$ stages, tumor location, differentiation grades and $\mathrm{N}$ stages between lymph node dissection group and non-dissection 

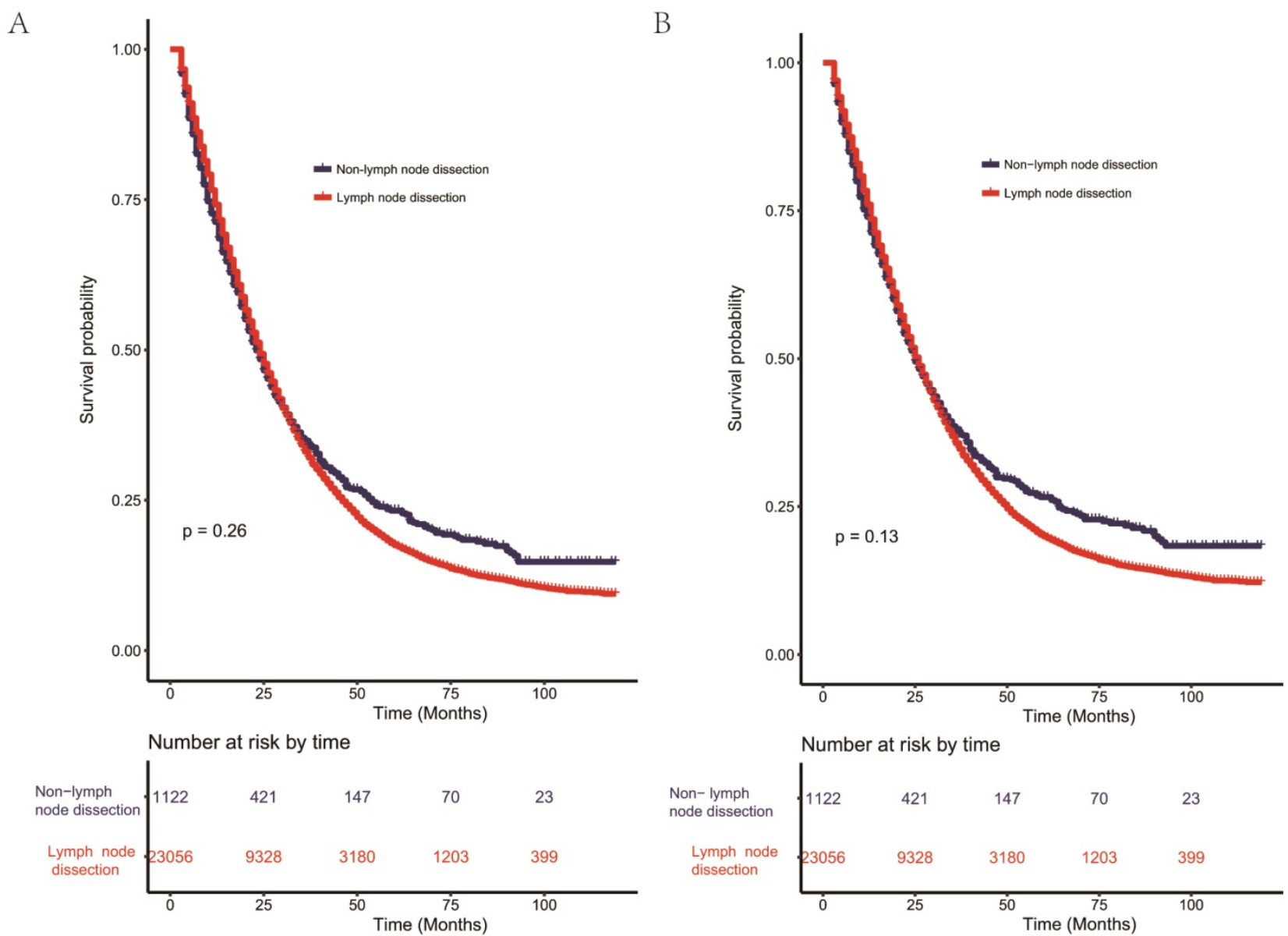

Figure 2. Kaplan-Meier curves for overall survival (A) and cause-specific survival (B) of lymph node dissection in resectable metastatic colorectal adenocarcinoma. Additional lymph node dissection for patients with resected primary metastatic colorectal adenocarcinoma showed poorer survival compared with non-lymph node dissection without statistical significance for overall survival $(P=0.26)$ and cause-specific survival $(P=0.13)$.

group. As shown in Table 4, patients with T1 stage of mCRC had better OS (HR: 0.51, 95\% CI: 0.39-0.66, $\mathrm{P}<0.001$ ) and CSS (HR: 0.48, 95\% CI: 0.36-0.65, $\mathrm{P}<0.001)$ if they received lymph node dissection, and patients with T3 stage had better OS (HR: 0.77, 95\% CI: $0.67-0.88, \mathrm{P}<0.001$ ) and CSS (HR: 0.76, 95\% CI: $0.66-0.87, \mathrm{P}<0.001)$ as well. As for location of tumor, patients with lymph node excision in proximal colon [OS (HR: 1.12, 95\% CI: 0.97-1.29, P=0.121) and CSS (HR: 1.11, 95\% CI: 0.95-1.29, $\mathrm{P}=0.177$ )] had no statistical difference with those without lymph node dissection. However, patients with distal adenocarcinoma [OS (HR: 0.65, 95\%CI: 0.56-0.75, $\mathrm{P}<0.001$ ) and CSS (HR: 0.65, 95\%CI: 0.56-0.75, $\mathrm{P}<0.001$ )] or rectal adenocarcinoma [OS (HR: 0.60, 95\% CI: $0.52-0.70, \mathrm{P}<0.001)$ and CSS (HR: 0.59, 95\% CI: 0.51-0.69, $\mathrm{P}<0.001)$ ] benefited from lymph node dissection. Patients with well or moderately differentiated disease had better OS (HR:0.62, 95\% CI: 0.56-0.70, $\mathrm{P}<0.001$ ) and CSS (HR: 0.62, 95\%CI: $0.55-0.69, \mathrm{P}<0.001$ ) after lymphadenectomy, while patients with poorly differentiated or undifferentiated disease [OS (HR: 1.04, 95\% CI: 0.91-1.18, P= 0.573) and
CSS (HR: 1.03, 95\% CI: 0.90-1.18, P= 0.652)] couldn't obtain a benefit from lymph node dissection. Patients with lymph node dissection could profit from survival in N1 [OS (HR: 0.76, 95\%CI: 0.67-0.85, $\mathrm{P}<0.001$ ) and CSS (HR: 0.74, 95\%CI: 0.65-0.84, $\mathrm{P}<0.001)$ ] and N2 [OS (HR: 0.63, 95\%CI: 0.54-0.74, $\mathrm{P}<0.001$ ) and CSS (HR: 0.65, 95\%CI: 0.55-0.77, $\mathrm{P}<0.001)$ ] stages, whereas with no benefit in N3 [OS (HR: 0.90, 95\% CI: 0.72-1.13, P=0.358) and CSS (HR: $0.89,95 \%$ CI: $0.71-1.13, \mathrm{P}=0.352)$ ] and N4 [OS (HR: 0.84, 95\% CI: 0.55-1.30, $\mathrm{P}=0.440$ ) and CSS (HR: 0.88, 95\% CI: 0.56-1.37, $\mathrm{P}=0.564)]$.

\section{Discussion}

In this study, we evaluated lymph node dissection as an independent protective factor for poor survival of patients with mCRC. According to our findings, for patients with $\mathrm{mCRC}$, lymph node dissection was suggested for patients with T1 stage and T3 stage. Also it was meaningless to perform lymph node dissection patients with stage T2 and T4. Moreover, patients with different location of tumors varied in OS and CSS when receiving lymph node 
dissection. Patients undergoing lymph node dissection benefited from lymphadenectomy with tumor of distal colon and rectum. For differentiation grades, lymph node dissection improved survival for patients with well or moderately differentiated adenocarcinoma, whereas lymph node dissection tended to make no difference in survival for patients with poorly differentiated or undifferentiated mCRC. For $\mathrm{N}$ stages, patients only in $\mathrm{N} 1$ and $\mathrm{N} 2$ of mCRC could benefit from lymphadenectomy.

Table 3. Multivariate Cox regression analysis for evaluating the influence of clinicalpathological characteristics on survival of patients with primary metastatic colorectal adenocarcinoma resection in SEER database.

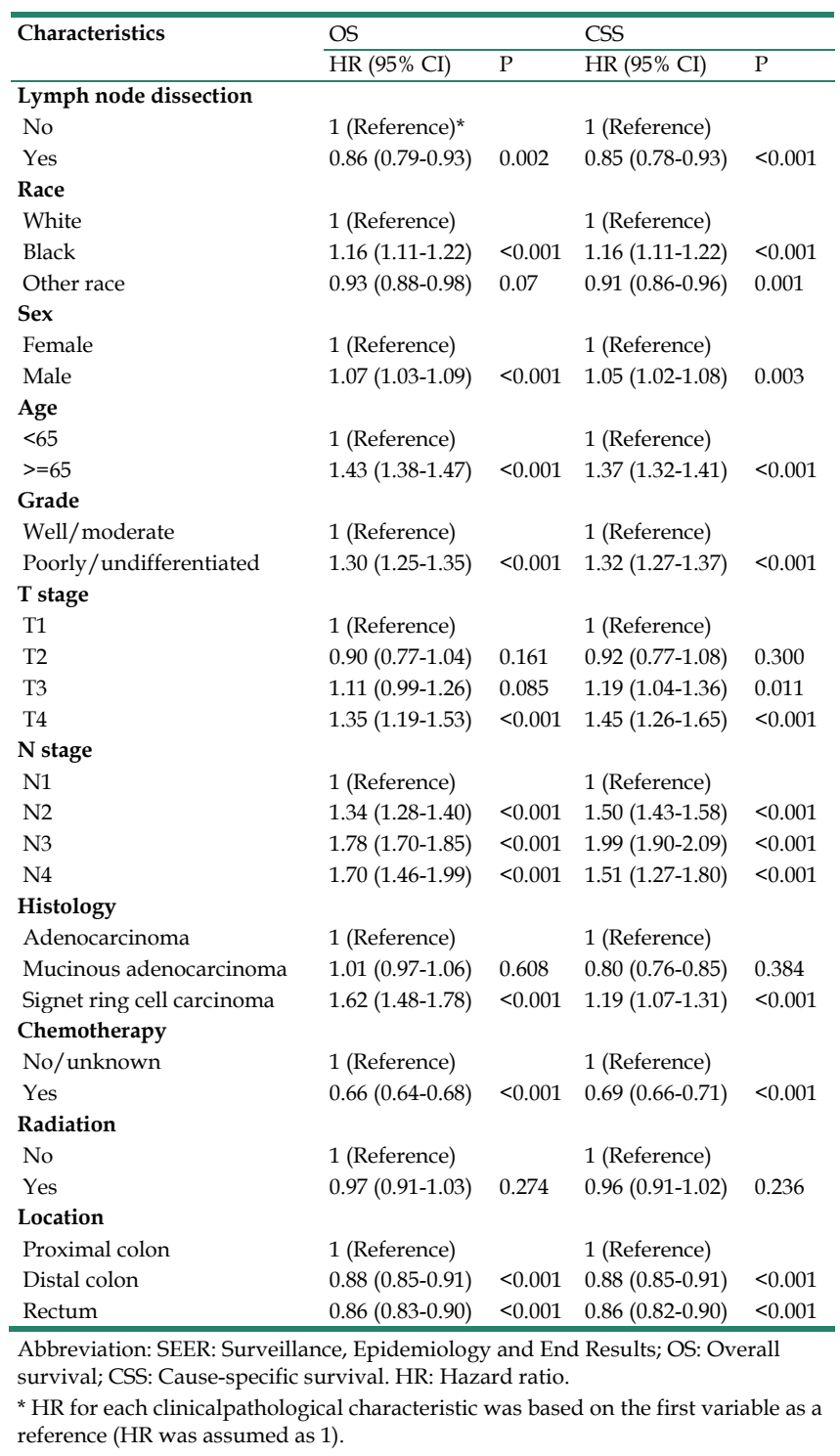

The treatment of patients with resectable stage IV CRC should be individualized and comprehensively multidisciplinary. When mCRC is regarded as resectable, resection of primary lesion should be performed following the principle of surgery [3]. According to NCCN Guidelines of colon and rectal cancer Version1.2017, at least 12 lymph nodes needed to be examined and clinically suspicious nodes should be removed if possible [7]. But, there was still an absence of precise guidance on whether lymph node dissection was needed on the matter of resectable mCRC. This study was the first study based on SEER database to assess the prognostic effect of lymph node dissection on survival of patients with $\mathrm{mCRC}$.

Table 4. Multivariate cox regression analysis of clinicalpathological characteristics on survival of patients with primary metastatic colorectal adenocarcinoma resection according to different $\mathrm{T}$ stages, $\mathrm{N}$ stages, tumor location or differentiation grades.

\begin{tabular}{|c|c|c|c|c|}
\hline \multirow[t]{2}{*}{ Characteristics } & \multicolumn{2}{|l|}{ OS } & \multicolumn{2}{|l|}{ CSS } \\
\hline & HR $(95 \%$ CI $)$ & $\mathrm{P}$ & HR $(95 \%$ CI $)$ & $\mathrm{P}$ \\
\hline \multicolumn{5}{|l|}{ T Stage } \\
\hline \multicolumn{5}{|l|}{ T1 } \\
\hline No & 1 (Reference)* & & 1 (Reference) & \\
\hline Yes & $0.51(0.39-0.66)$ & $<0.001$ & $0.48(0.36-0.65)$ & $<0.001$ \\
\hline \multicolumn{5}{|l|}{$\mathrm{T} 2$} \\
\hline No & 1 (Reference) & & 1 (Reference) & \\
\hline Yes & $1.09(0.71-1.68)$ & 0.512 & $0.93(0.60-1.44)$ & 0.740 \\
\hline \multicolumn{5}{|l|}{ T3 } \\
\hline No & 1 (Reference) & & 1 (Reference) & \\
\hline Yes & $0.77(0.67-0.88)$ & $<0.001$ & $0.76(0.66-0.87)$ & $<0.001$ \\
\hline \multicolumn{5}{|l|}{$\mathrm{T} 4$} \\
\hline No & 1 (Reference) & & 1 (Reference) & \\
\hline Yes & $0.96(0.84-1.10)$ & 0.542 & $0.97(0.85-1.12)$ & 0.715 \\
\hline \multicolumn{5}{|l|}{ Location } \\
\hline \multicolumn{5}{|l|}{ Proximal colon } \\
\hline No & 1 (Reference) & & 1 (Reference) & \\
\hline Yes & $1.12(0.97-1.29)$ & 0.121 & $1.11(0.95-1.29)$ & 0.177 \\
\hline \multicolumn{5}{|l|}{ Distal colon } \\
\hline No & 1 (Reference) & & 1 (Reference) & \\
\hline Yes & $0.65(0.56-0.75)$ & $<0.001$ & $0.65(0.56-0.75)$ & $<0.001$ \\
\hline \multicolumn{5}{|l|}{ Rectum } \\
\hline No & 1 (Reference) & & 1 (Reference) & \\
\hline Yes & $0.60(0.52-0.70)$ & $<0.001$ & $0.59(0.51-0.69)$ & $<0.001$ \\
\hline \multicolumn{5}{|l|}{ Grade } \\
\hline \multicolumn{5}{|l|}{ Well/moderate } \\
\hline No & 1 (Reference) & & 1 (Reference) & \\
\hline Yes & $0.62(0.56-0.70)$ & $<0.001$ & $0.62(0.55-0.69)$ & $<0.001$ \\
\hline \multicolumn{5}{|c|}{ Poorly/undifferentiated } \\
\hline No & 1 (Reference) & & 1 (Reference) & \\
\hline Yes & $1.04(0.91-1.18)$ & 0.573 & $1.03(0.9-1.18)$ & 0.652 \\
\hline \multicolumn{5}{|l|}{ N Stage } \\
\hline \multicolumn{5}{|l|}{ N1 } \\
\hline No & 1 (Reference) & & 1 (Reference) & \\
\hline Yes & $0.76(0.67-0.85)$ & $<0.001$ & $0.74(0.65-0.84)$ & $<0.001$ \\
\hline \multicolumn{5}{|l|}{ N2 } \\
\hline No & 1 (Reference) & & 1 (Reference) & \\
\hline Yes & $0.63(0.54-0.74)$ & $<0.001$ & $0.65(0.55-0.77)$ & $<0.001$ \\
\hline \multicolumn{5}{|l|}{ N3 } \\
\hline No & 1 (Reference) & & 1 (Reference) & \\
\hline Yes & $0.90(0.72-1.13)$ & 0.358 & $0.89(0.71-1.13)$ & 0.352 \\
\hline \multicolumn{5}{|l|}{ N4 } \\
\hline No & 1 (Reference) & & 1 (Reference) & \\
\hline Yes & $0.84(0.55-1.30)$ & 0.440 & $0.88(0.56-1.37)$ & 0.564 \\
\hline
\end{tabular}

So far as we knew, there were some studies carried out to identify the prognostic effect of lymph 
node dissection for stage I to III but not for stage IV. A cohort study from The United Kingdom revealed that lymph node retrieval had no significant effect on OS for Dukes stages A to C of colon cancer [15]. Kobayashi et al. found it unnecessary for patients with well-differentiated adenocarcinoma to remove regional lymph node after endoscopic resection in $\mathrm{T} 1$ colorectal cancer [16]. A retrospective study in 2017 indicated that patients who had more lymph node dissected seemed to promote survival, not relying on stage [17]. As opinions varied, no unanimous conclusion can be drawn. To date, there has been no direct evidence of relationship between lymph node dissection and survival of patients with resectable mCRC. Thus, our study was unique that provided a population-based research evidence of the role of lymph node excision in survival of patients with mCRC.

As shown in the Figure 2, additional lymph node dissection for patients with resected primary metastatic colorectal adenocarcinoma showed poorer survival compared with non-lymph node dissection without statistical significance for overall survival $(\mathrm{P}=$ $0.26)$ and cause-specific survival $(P=0.13)$, which indicated some confounding factors may affect the influence of lymph node dissection on survival of patients with mCRC. Further analysis using univariate and multivariate Cox analysis verified our conclusion. Though no difference existed between two groups in the univariate Cox model, multivariate Cox analysis showed lymph node dissection improved survival of patients with mCRC.

For T1 stage of $\mathrm{mCRC}$, the tumor burden of cancer can be relieved to some extent by neoadjuvant chemotherapy, resection of the primary tumor, dissection of lymph node and excision of metastases. When it comes to tumor' penetration to the surface of the visceral peritoneum (T4a) or tumor's direct invasion or adhesion to other organs (T4b), excision of lymph node may not be suggested. One possible reason was that patients were too weak to receive tumor radical surgery with regional lymph node dissection.

As we all knew, published literature had defined colorectal carcinoma into three segments of the gut: proximal colon, distal colon and rectum. Proximal CRC is located within the proximal two-thirds of transverse colon, ascending colon and caecum, derived from the embryologic midgut. While distal colorectal carcinoma is seated in the colon derived from the embryologic hindgut, including distal third of the transverse colon, splenic flexure, descending colon, sigmoid colon and rectum [18]. Differences were noted in clinical characteristics, epidemiology, molecular feature, surgical treatment and clinical outcome due to different location of CRC $[18,19]$. According to a retrospective, population-based study, proximal colon carcinoma presented at more advanced stage than distal colon and rectal cancer [19, 20]. And there was a higher percent of proximal colon carcinoma dissected with node-positive disease than distal colorectal cancer [20]. In general, previous studies only mentioned that proximal colon cancer had more positive node invasion and worse outcome, but there was no evidence on whether lymph node should be removed. Our study gave direct suggestion to surgeon that lymph node excision could be performed for distal colorectal cancer of metastatic patients but may not for proximal colon cancer patients.

Likewise, lymphadenectomy would not be advised for patients with poorly or undifferentiated cancer disease. Patients with poorly or undifferentiated cancer usually had more progressive diseases and more positive nodes involved [21], so that they are unable to withstand extra lymph excision. Besides, according to survival analysis of $\mathrm{N}$ stages in lymph node dissection group and non-dissection group, lower lymph node involvement (N1 and N2) were associated with improved survival when receiving lymphadenectomy for patients with mCRC. While for patients in N3 and N4 stage who could not tolerate operation, adjuvant therapy might be the better.

We should acknowledge some limitations in this study. Firstly, any retrospective study succumbs to inherent bias. The role of colorectal carcinoma excision and lymphadenectomy in patients with mCRC cannot be completely illuminated without a prospective and randomized clinical trial. Secondly, the detailed information on chemotherapy such as neoadjuvant chemotherapy, chemotherapy regimens, which were also related to the survival after lymph node dissection, was not available in the SEER database. Also, response to chemotherapy was associated with genetic status such as KRAS, NRAS, BRFA, and MSI, which was not available in SEER database. There had been studies regarding association of poor survival of right colon cancer and those studies revealed poor response to chemotherapy in proximal colorectal cancer was caused by different genetic status between locations of tumor $[18,22]$. Likewise, lacking of information on metastatic extent, metastatic disease burden and complication rates of surgery led to misjudging the value of lymphadenectomy. The above mentioned factors should be considered in next potential analysis on whether lymphadenectomy improved surivial of patietns with resectable mCRC especially mCRC of $\mathrm{T} 4$, poor or undifferentiation, N3/N4 stage or 
proximal colon. Thirdly, quite number of patients with survival time less than three months were excluded in this analysis, which could also cause bias in analysis. However, death during three months were mostly attributed to complication of surgery and patient's condition and could not reveal clinical significance of lymphadenectomy to resectable mCRC. Fourthly, the information of simultaneous or metachronous metastases, which was a risk factor for poor survival of $\mathrm{mCRC}$, was not available for cases included in this study. Finally, all the findings and conclusions were derived from the pre-existing database and we failed to validate our finding in our own institute, which caused relatively low reliability of our conclusions without external validation. Therefore, the results should be explained with caution and further study should be performed to validate our findings and conclusions.

\section{Conclusion}

In general, our findings revealed that a significant improvement in survival resulted from lymph node dissection and metastatic colorectal adenocarcinoma resection of patients with T1 stage, well or moderately differentiated, N1and N2 stage, and distal or rectal cancer. However, additional lymph node dissection may not be necessary for patients with primary $\mathrm{mCRC}$ dissection of $\mathrm{T} 4$, poor or undifferentiation, N3 and N4 stage and proximal colon. Further research ought to be conducted to confirm the findings above.

\section{Acknowledgements}

Thanks for all the datasets provided by the SEER Program and we obey the provisions of SEER. This study was funded by the project of Wenzhou Municipal Science and Technology Bureau (Grant number Y20170180).

\section{Competing Interests}

The authors have declared that no competing interest exists.

\section{References}

1. Siegel RL, Miller KD, Jemal A. Cancer Statistics, 2017. CA Cancer J Clin.2017; 67: 7-30.

2. Pfister DG, Benson AB 3rd, Somerfield MR . Clinical practice. Surveillance strategies after curative treatment of colorectal cancer. N Engl J Med. 2004; 350: 2375-2382

3. Vogel JD, Eskicioglu C, Weiser MR, et al. The American Society of Colon and Rectal Surgeons Clinical Practice Guidelines for the Treatment of Colon Cancer. Dis Colon Rectum. 2017; 60: 999-1017.

4. Reddy SK, Pawlik TM, Zorzi D, et al. Simultaneous resections of colorectal cancer and synchronous liver metastases: a multi-institutional analysis. Ann Surg Oncol. 2007; 14: 3481-3491.

5. Yamazaki M, So T, Mizukami M, et al. 2002. Pulmonary resection for metastatic colorectal cancer. J UOEH. 2002; 24: 189-195.

6. Kidner TB, Ozao-Choy JJ, Yoon J, et al. Should quality measures for lymph node dissection in colon cancer be extrapolated to rectal cancer? Am J Surg. 2012; 204: 843-847.
7. Le Voyer TE, Sigurdson ER, Hanlon AL, et al. Colon cancer survival is associated with increasing number of lymph nodes analyzed: a secondary survey of intergroup trial INT-0089. J Clin Oncol. 2003; 21: 2912-2919.

8. Lee WS, Yun SH, Chun HK, et al. Pulmonary resection for metastases from colorectal cancer: prognostic factors and survival. Int J Colorectal Dis. 2007; 22 : $699-704$.

9. Van Cutsem E, Nordlinger B, Adam R, et al. Towards a pan-European consensus on the treatment of patients with colorectal liver metastases. Eur J Cancer. 2006; 42: 2212-2221.

10. Yoo PS, Lopez-Soler RI, Longo WE, et al. Liver resection for metastatic colorectal cancer in the age of neoadjuvant chemotherapy and bevacizumab. Clin Colorectal Cancer . 2006; 6: 202-207.

11. Ardito F, Vellone M, Cassano A, et al. Chance of cure following liver resection for initially unresectable colorectal metastases: analysis of actual 5-year survival. J Gastrointest Surg. 2013; 17: 352-359.

12. Zhang $\mathrm{CH}$, Pan $\mathrm{YB}$, Zhang QW, et al. The influence of local therapy on the survival of patients with metastatic rectal cancer: a population-based, propensity-matched study. J Cancer Res Clin Oncol. 2017.

13. Pindak D, Pavlendova J, Tomas $M$, et al. Selective versus routine lymphadenectomy in the treatment of liver metastasis from colorectal cancer: a retrospective cohort study. BMC Surg 2017; 17: 34.

14. Bradatsch A, Kornprat $\mathrm{P}$, Bacher $\mathrm{H}$, et al. The Value of Lymph Node Dissection in the Surgery of Colorectal Cancer Liver Metastases. Anticancer Res. 2016; 36: 2993-2997.

15. Rao A, Dadras M, Razzak MA, et al. Effect of Lymph Node Retrieval and Ratio on the Long-Term Survival and Recurrence of Colon Cancer. J Coll Physicians Surg Pak. 2016; 26: 467-470.

16. Kobayashi $\mathrm{H}$, Higuchi $\mathrm{T}$, Uetake $\mathrm{H}$, et al. Resection with en bloc removal of regional lymph node after endoscopic resection for T1colorectal cancer. Ann Surg Oncol. 2012; 19: 4161-4167.

17. O'Boyle S, Stephenson K. More is better: Lymph node harvesting in colorectal cancer. Am J Surg. 2017; 213: 926-930.

18. Stintzing $\mathrm{S}$, Tejpar $\mathrm{S}$, Gibbs $\mathrm{P}$, et al. Understanding the role of primary tumour localisation in colorectal cancer treatment and outcomes. Eur J Cancer. 2017; 84: 69-80.

19. Snaebjornsson P, Jonasson L, Jonsson $\mathrm{T}$, et al. Colon cancer in Iceland--a nationwide comparative study on various pathology parameters with respect to right and left tumor location and patients age. Int J Cancer. 2010; 127: 2645-2653.

20. Meguid RA, Slidell MB, Wolfgang CL, et al. Is there a difference in survival between right- versus left-sided colon cancers? Ann Surg Oncol. 2008; 15: 2388-2394.

21. Hong M, Kim JW, Shin MK, et al. Poorly Differentiated Clusters in Colorectal Adenocarcinomas Share Biological Similarities with Micropapillary Patterns as well as Tumor Buds. J Korean Med Sci. 2017; 32: 1595-1602.

22. Hong SP, Min BS, Kim TI, et al. The differential impact of microsatellite instability as a marker of prognosis and tumour response between colon cancer and rectal cancer. Eur J Cancer. 2012; 48: 1235-1243. 